\title{
Terapia vocal no contexto da Telefonoaudiologia em pacientes disfônicos: revisão integrativa
}

\section{Voice therapy in the context of Telespeech therapy in dysphonics}

\author{
patients: an integrative review
}

\author{
Mariana Rebeka Gomes Queiroz ${ }^{1}$ (D), Leandro Araújo Pernambuco² (D), Rebeca Lins de Souza Leão ${ }^{1}$ (D), \\ Jonia Alves Lucena' ${ }^{1}$ (D)
}

\section{RESUMO}

Objetivos: Descrever as características da terapia vocal por meio da Telefonoaudiologia com pacientes disfônicos. Estratégia de pesquisa: A pesquisa foi baseada na metodologia PCC (População/Conceito/Contexto) e envolveu a elaboração da questão a ser investigada, localização e seleção de estudos, extração de dados e avaliação crítica dos trabalhos, segundo protocolo Joanna Briggs. A busca dos estudos foi realizada nas bases BVS, PubMed/MEDLINE, Web of Science, Scopus e Embase, nos idiomas inglês, português e espanhol, por meio dos descritores do DeCS, MeSH e Emtree, entre os operadores booleanos. Critérios de seleção: foram incluídos estudos originais, sem limite de ano de publicação, que envolvessem $\mathrm{o}$ atendimento à população disfônica, por Telefonoaudiologia. Foram excluídas as publicações repetidas nas bases de dados. Resultados: Foram encontrados 5.740 estudos, dos quais apenas quatro artigos foram incluídos após os critérios de elegibilidade. As publicações foram registradas entre 2015 e 2020, com maior número nos Estados Unidos da América. A maioria dos trabalhos foi realizada com mulheres idosas. A intervenção apresentou variações quanto ao método de funcionamento, segurança de dados, número, frequência e duração de sessões, além de exercícios terapêuticos. Ademais, foram apontadas melhorias em diversos parâmetros vocais avaliados nos estudos. Conclusão: A Telefonoaudiologia junto a pacientes disfônicos é voltada para diferentes públicos com etiologias vocais diversas. Além disso, há diferentes metodologias empregadas para o atendimento em voz à distância, com resultados positivos relacionados aos parâmetros vocais, bem como satisfação do paciente quanto ao tratamento realizado nesse formato.

Palavras-chave: Voz; Disfonia; Distúrbios da voz; Treinamento da voz; Telerreabilitação

\begin{abstract}
Purpose: To describe the characteristics of telespeech therapy for dysphonic patients. Research strategy: The research was based on the PCC method (Population/Concept/Context) and involved developing the research question, finding and selecting the studies, extracting the data, and clinically assessing the papers, based on Joanna Briggs protocol. The studies were searched in VHL, PubMed/MEDLINE, Web of Science, Scopus, and EMBASE in English, Portuguese, and Spanish, with descriptors from DeCS, MeSH, and Emtree and using the Boolean operators. Selection criteria: Original studies addressing telespeech therapy for the dysphonic population were included, with no restriction of publication year. Duplicate publications in the databases were excluded. Results: A total of 5,740 studies were found, of which only four were included based on the eligibility criteria. The studies were published between 2015 and 2020, and most of them were from the United States. Most studies were carried out with older women. The intervention had a variety of functioning methods, data safety, number, frequency, and duration of the sessions, and therapeutic exercises. There were improvements in various voice parameters assessed in the studies. Conclusion: Telespeech therapy for dysphonic patients is aimed at different audiences with different voice problem etiologies. Various methodologies were employed in remote voice healthcare with positive voice parameter results and the patients' satisfaction with the treatment they received in this format.
\end{abstract}

Keywords: Voice; Dysphonia; Voice disorders; Voice training; Telerehabilitation

Trabalho realizado na Universidade Federal de Pernambuco - UFPE - Recife (PE), Brasil.

${ }^{1}$ Programa de Pós-graduação (Mestrado) em Saúde da Comunicação Humana, Universidade Federal de Pernambuco - UFPE - Recife (PE), Brasil.

${ }^{2}$ Programa Associado de Pós-graduação em Fonoaudiologia, Universidade Federal da Paraíba-UFPB-João Pessoa (PB), Brasil

Conflito de interesses: Não.

Contribuição dos autores: MRGQ, LAP e JAL elaboraram a concepção e delineamento do estudo; MRGQ, RLSL, LAP e JAL realizaram análise e interpretação dos dados, revisão crítica intelectual e aprovaram a versão final a ser publicada.

Financiamento: Coordenação de Aperfeiçoamento de Pessoal de Nível Superior (CAPES), Número do contrato 001.

Autor correspondente: Mariana Rebeka Gomes Queiroz. E-mail: marianaqueiroz@hotmail.com

Recebido: Junho 02, 2021; Aceito: Outubro 27, 2021 


\section{INTRODUÇÃO}

$\mathrm{O}$ atendimento a distância na Fonoaudiologia, a Telefonoaudiologia, é uma prática regulamentada, anteriormente realizada em contextos terapêuticos específicos. Tal prática era voltada para os pacientes com dificuldade no acesso ao profissional $^{(1)}$ e também como medida de custo-benefício quanto à redução no tempo de viagem dos terapeutas e/ou pacientes ${ }^{(1,2)}$ e no deslocamento de indivíduos com mobilidade física prejudicada ${ }^{(3)}$.

No contexto da pandemia da Covid-19, a Telefonoaudiologia entrou em processo de expansão, especialmente devido à necessidade de distanciamento e isolamento social. Apesar de a Telefonoaudiologia ter sido regulamentada pelo Conselho de Federal de Fonoaudiologia em $2013^{(4)}$, não era utilizada em massa, como está ocorrendo durante a pandemia ${ }^{(5,6)}$. Assim, diante do cenário de saúde mundial, foi necessária a reestruturação da oferta de serviço, com afirmação do atendimento virtual por meio da nova regulamentação da Telefonoaudiologia, em $2020^{(7)}$.

Para o atendimento a distância, algumas barreiras são encontradas. Na maioria dos casos, estão relacionadas à falta de acesso dos pacientes a recursos tecnológicos, ao fato de não estarem dispostos a utilizar esse novo formato de atendimento, ou mesmo à preferência pela realização do atendimento presencial. Em relação à adesão por profissionais, é possível encontrar dificuldades de aceitação e aplicabilidade por falta de manejo da ferramenta virtual, ou, ainda, pela ocorrência de baixa qualidade de imagem/áudio/internet, o que pode levar à descontinuação da sessão ${ }^{(1-3,8)}$.

Especificamente na área de voz, existem dificuldades adicionais relacionadas à necessidade de avaliação presencial e utilização de softwares com alta qualidade de vídeo e gravação de áudio com calibração por meio de um processador de fala acústica $^{(9)}$. Mesmo diante das dificuldades nessa área, chama-se a atenção para uma demanda prevalente voltada aos pacientes com Parkinson, com registro de resultados positivos no póstratamento, principalmente quanto à utilização do método Lee Silverman- LSVT ${ }^{\left({ }^{(10-12)}\right.}$ na Telefonoaudiologia. Ainda em ambiente virtual, afora a aplicação do LSVT, na clínica vocal também são utilizadas outras metodologias, como orientação vocal $^{(13,14)}$, terapia de voz ressoante, exercício de função vocal, relaxamento e tarefas de fala direcionada ${ }^{(14)}$. De forma geral, os diversos estudos destacam melhorias na autopercepção vocal ${ }^{(15)}$, no tempo máximo de fonação ${ }^{(14)}$, bem como nos parâmetros acústicos vocais, com destaque para o nível de pressão sonora ${ }^{(11,12)}$, além dos parâmetros perceptivo-auditivos, a exemplo do grau geral da disfonia ${ }^{(14,15)}$, rugosidade $^{(14)}$, astenia ${ }^{(14)}$ e loudness ${ }^{(10,12)}$ Portanto, percebe-se que essa modalidade de reabilitação vocal a distância pode beneficiar os pacientes com disfonia.

A presente pesquisa questiona: "Quais as características da terapia vocal por meio da Telefonoaudiologia com pacientes disfônicos?" Supõe-se que a Telefonoaudiologia seja uma modalidade adicional ao atendimento do paciente disfônico, que envolve a possibilidade de uso de metodologias diversas, com resultados positivos e satisfação do usuário.

Considerando que essa modalidade de atendimento está em crescimento e diante das atuais necessidades da Telefonoaudiologia, preservando-se a segurança e saúde das pessoas, faz-se necessário conhecer melhor as experiências de terapia vocal nesse formato. O presente estudo buscou sintetizar o conhecimento científico sobre as características desse formato na prática clínica em voz. Almejou-se apontar evidências para que o fonoaudiólogo clínico possa atuar de forma segura quanto ao atendimento a distância. Esta revisão possibilitou, ainda, contribuir para a criação de estratégias para promoção, avaliação, monitoramento e terapia vocal na Telefonoaudiologia.

\section{OBJETIVO}

Descrever as características da terapia vocal por meio da Telefonoaudiologia com pacientes disfônicos.

\section{Estratégia de pesquisa}

Esta revisão foi construída de acordo com as seguintes etapas: (1) construção da pergunta norteadora; (2) elaboração dos critérios de elegibilidade e busca na literatura; (3) extração dos dados dos artigos selecionados; (4) avaliação crítica dos estudos; (5) interpretação/discussão dos achados; (6) apresentação da revisão integrativa ${ }^{(16)}$.

A pergunta norteadora foi "Quais as características da terapia vocal por meio da Telefonoaudiologia com pacientes disfônicos?". A pergunta foi baseada nas estratégias do PCC, sendo $\mathrm{P}$ (População) = indivíduos com disfonia; $\mathrm{C}$ (Conceito) $=$ terapia vocal $; \mathrm{C}($ Contexto $)=$ Telefonoaudiologia ${ }^{(17)}$.

Para responder a esse questionamento, foram pesquisados artigos originais nas bases de dados: BVS, PubMed/MEDLINE, Web of Science, Scopus e Embase, por meio dos descritores em inglês, espanhol e português, selecionados do DeCS (Descritores em Ciências da Saúde), MeSH (Medical Subject Headings) e Emtree (Embase Subject Headings). A coleta ocorreu de 28 de janeiro de 2021 a 20 de fevereiro de 2021. Os descritores utilizados foram: em inglês: Dysphonia OR "voice disorder" AND "voice training" AND telerehabilitation AND voice; em espanhol: Disfonía OR "transtornos de la voz" AND "entrenamiento de la voz" AND telereabilitación AND voz; em português: Disfonia OR distúrbios da voz AND treinamento da voz AND telerreabilitação AND voz. As estratégias estão descritas no Quadro 1.

Quadro 1. Bases de dados pesquisadas e suas respectivas estratégias de busca empregadas

\begin{tabular}{|ll|}
\hline \multicolumn{1}{|c|}{ Bases } & \multicolumn{1}{c|}{ Estratégias de busca } \\
\hline PubMed & ((dysphonia OR "voice disorder") AND "voice training" AND telerehabilitation AND voice)) \\
BVS & ((dysphonia OR "voice disorder") AND "voice training" AND telerehabilitation AND voice)) \\
BVS & disfonía OR "Trastornos de la Voz" AND "Entrenamiento de la Voz" AND Telerehabilitación AND voz \\
BVS & disfonia OR "Distúrbios da Voz" AND "Treinamento da Voz" AND Telerreabilitação AND voz \\
Scopus & dysphonia OR "voice disorder" AND "voice training " AND telerehabilitation AND voice \\
Embase & dysphonia OR voice disorder AND voice training AND telerehabilitation AND voice \\
Web of Science & dysphon* OR voice disord
\end{tabular}


A busca na literatura foi baseada em artigos que envolvessem unicamente a terapia vocal em disfônicos, por meio da Telefonoaudiologia. Assim, não foram excluídos os termos amplos, a exemplo de telemedicina, telessaúde e telemonitoramento.

A identificação dos artigos foi realizada por dois revisores independentes, por meio da leitura inicial do título, aplicando os critérios de inclusão ou exclusão, e também leitura do resumo. Por fim, foram lidos os artigos na íntegra. Tendo em vista as discordâncias entre os dois revisores iniciais, um terceiro revisor foi solicitado para participar da análise dos artigos. Sua participação foi considerada apenas no julgamento de artigos com discrepância entre os dois primeiros revisores.

\section{Critérios de seleção}

Os critérios de inclusão foram: artigos originais (transversais, caso-controle, coorte, quase experimental e os ensaios clínicos randomizados), sem limite de ano de publicação, que envolvessem $\mathrm{o}$ atendimento à população disfônica por Telefonoaudiologia. Foram excluídas publicações repetidas nas bases de dados.

\section{Análise dos dados}

A extração e o gerenciamento de dados foram conduzidos por uma planilha eletrônica elaborada no Excel e preenchida pelos revisores. Para cada estudo incluído, foram analisados e extraídos os seguintes dados: autor/ano; local do estudo; desenho; objetivos; população (faixa etária, número de sujeitos, sexo); método (método de funcionamento do atendimento a distância; segurança de dados; recursos utilizados, número de sessões, tipo de exercício); resultados.

A avaliação crítica dos estudos consistiu na análise da qualidade metodológica dos artigos selecionados. Esta fase foi realizada por dois revisores, baseada nos protocolos do Joanna Briggs Institute (JBI) ${ }^{(18)}$ conforme o desenho do estudo. Em seguida, para a presente a revisão, só foram considerados como artigos inclusos aqueles com, no mínimo, $60 \%$ dos itens positivos nos protocolos do $\mathrm{JBI}^{(19)}$.

\section{RESULTADOS}

Dos 5.740 artigos encontrados nas bases de dados, 2 foram selecionados da PubMed, 8 da BVS, 4 da Scopus, 0 (zero) na Embase e 5.726 na Web of Science, porém, apenas 4 foram incluídos na revisão integrativa (Figura 1). Após a avaliação da qualidade metodológica, permaneceram os 4 trabalhos.

Os anos das publicações variaram de 2015 a 2020; o local de estudo predominante foram os Estados Unidos (50\%); os desenhos destacaram-se como quase experimental $(50 \%)$ e ensaio clínico $(50 \%)$; os objetivos, de forma geral, buscaram investigar as aplicações das sessões de terapia vocal a distância em indivíduos com etiologias diversas - doença de Parkinson (25\%); disfonia por tensão muscular $(50 \%)$ e idosos com alterações vocais (25\%). As amostras foram constituídas por um número que variou entre 8 e 69 sujeitos, com presença marcante para o sexo feminino e populações que incluíram desde o público jovem ao idoso, com destaque para o idoso. Sobre o Telefonoaudiologia em voz, foram encontrados trabalhos que realizavam apenas intervenção à distância ${ }^{(11,20)}$ ou pesquisas que faziam comparações entre grupos de Telefonoaudiologia e terapia convencional $^{(21,22)}$. Quanto à reabilitação, foram executadas, em sua maioria, no formato síncrono ${ }^{(11,21,22)}$. Sobre a segurança de dados, 2 artigos ${ }^{(11,20)}$ realizaram por meio do sistema SSL (Secure Sockets Layer) e Adobe Connect. As intervenções ocorreram em 8 a 12 sessões, com frequência de uma a duas vezes por semana, duração de 20 a 90 minutos e intervenções variadas (higiene vocal, método Lee Silverman - LSVT®, exercícios de fluxo de ar, exercício de trato vocal semiocluído, exercício de função vocal e exercício de controle percebido para os indivíduos com problemas de voz). A maioria dos achados dos estudos apontou melhorias nos parâmetros acústicos, perceptivo-auditivos, tempo máximo de fonação (TMF) e de autopercepção vocal, além da aceitabilidade e usabilidade do tratamento expressas pelo paciente nesse formato, com uso de recursos diferentes (software, aplicativo e plataforma de videoconferência). Os Quadros 2 e 3 demonstram as características dos estudos incluídos nesta revisão.

Quadro 2. Caracterização geral dos estudos incluídos

\begin{tabular}{|c|c|c|c|c|}
\hline Autor & Local & Tipo de estudo & Objetivo & Amostra \\
\hline Lin et al. ${ }^{(21)}$ & China & $\begin{array}{l}\text { Ensaio Clínico } \\
\text { Randomizado }\end{array}$ & $\begin{array}{l}\text { Examinar a hipótese de que a terapia vocal } \\
\text { via teleprática não é inferior à terapia vocal } \\
\text { tradicional. }\end{array}$ & $\begin{array}{l}69 \text { sujeitos distribuídos em dois grupos: } \\
\text { teleprática ( } 33 \text { participantes, sendo } 14 \\
\text { mulheres e } 19 \text { homens, faixa etária de } 57 \\
\text { a } 82 \text { anos e média de } 66 \text { anos) e terapia } \\
\text { convencional ( } 36 \text { participantes, sendo } 19 \\
\text { mulheres e } 17 \text { homens, com } 58 \text { a } 81 \text { anos } \\
\text { e média de } 69 \text { anos). }\end{array}$ \\
\hline Quinn et al.(11) & Austrália & $\begin{array}{c}\text { Quase- } \\
\text { experimental }\end{array}$ & $\begin{array}{l}\text { Determinar a viabilidade de um programa } \\
\text { de manutenção de fala para pessoas } \\
\text { com doença de Parkinson, por meio da } \\
\text { telerreabilitação. }\end{array}$ & $\begin{array}{l}8 \text { sujeitos com doença de Parkinson, } 2 \\
\text { mulheres e } 6 \text { homens, } 61 \text { a } 81 \text { anos e } \\
\text { média de } 69 \text { anos. }\end{array}$ \\
\hline Nguyen-Feng et al. ${ }^{(20)}$ & EUA & $\begin{array}{c}\text { Quase- } \\
\text { experimental }\end{array}$ & $\begin{array}{l}\text { Estabelecer a viabilidade, usabilidade e } \\
\text { aceitabilidade de uma intervenção online } \\
\text { de controle percebido para indivíduos com } \\
\text { distúrbios de voz e coletar dados preliminares } \\
\text { sobre a eficácia da intervenção. }\end{array}$ & $\begin{array}{l}20 \text { disfônicos distribuídos em dois } \\
\text { grupos: } 10 \text { universitários com disfonia } \\
\text { por tensão muscular e } 10 \text { sujeitos que se } \\
\text { autodeclararam disfônicos; } 12 \text { mulheres e } \\
8 \text { homens, } 18 \text { a } 80 \text { anos. }\end{array}$ \\
\hline Rangarathnam et al.(22) & EUA & $\begin{array}{l}\text { Prospectivo } \\
\text { Randomizado }\end{array}$ & $\begin{array}{l}\text { Determinar a utilidade da teleprática para } \\
\text { realização de exercícios de fonação em } \\
\text { fluxo para pessoas com disfonia por tensão } \\
\text { muscular primária. }\end{array}$ & $\begin{array}{l}14 \text { indivíduos com disfonia por tensão } \\
\text { muscular, divididos em dois grupos: } \\
\text { teleprática (7) e terapia convencional (7); } \\
11 \text { mulheres e } 3 \text { homens, } 16 \text { a } 81 \text { anos e } \\
\text { média de } 50 \text { anos. }\end{array}$ \\
\hline
\end{tabular}

Legenda: $\mathrm{EUA}=$ Estados Unidos da América 

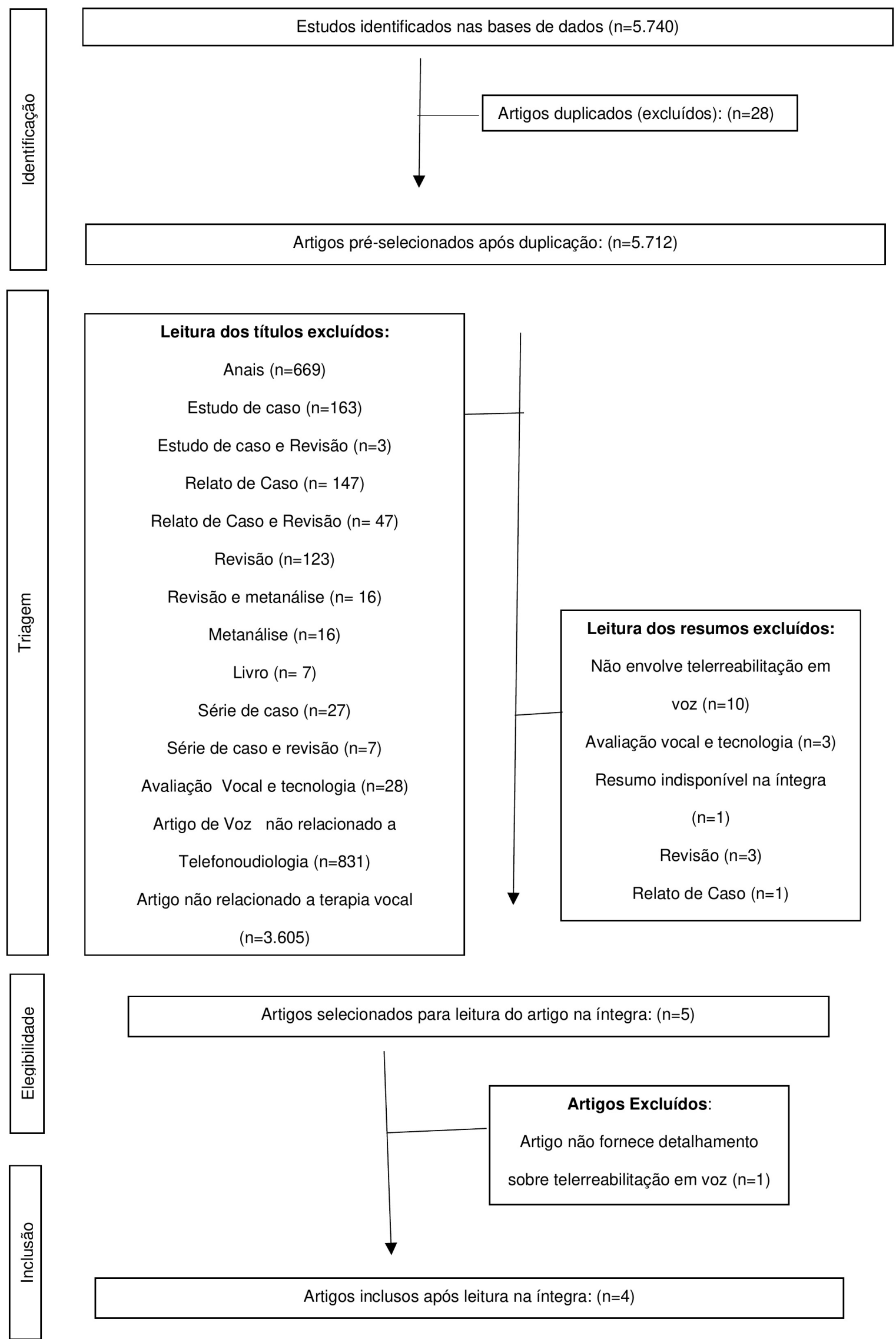

Figura 1. Fluxograma de seleção dos estudos Legenda: $\mathrm{n}=$ número de estudos 


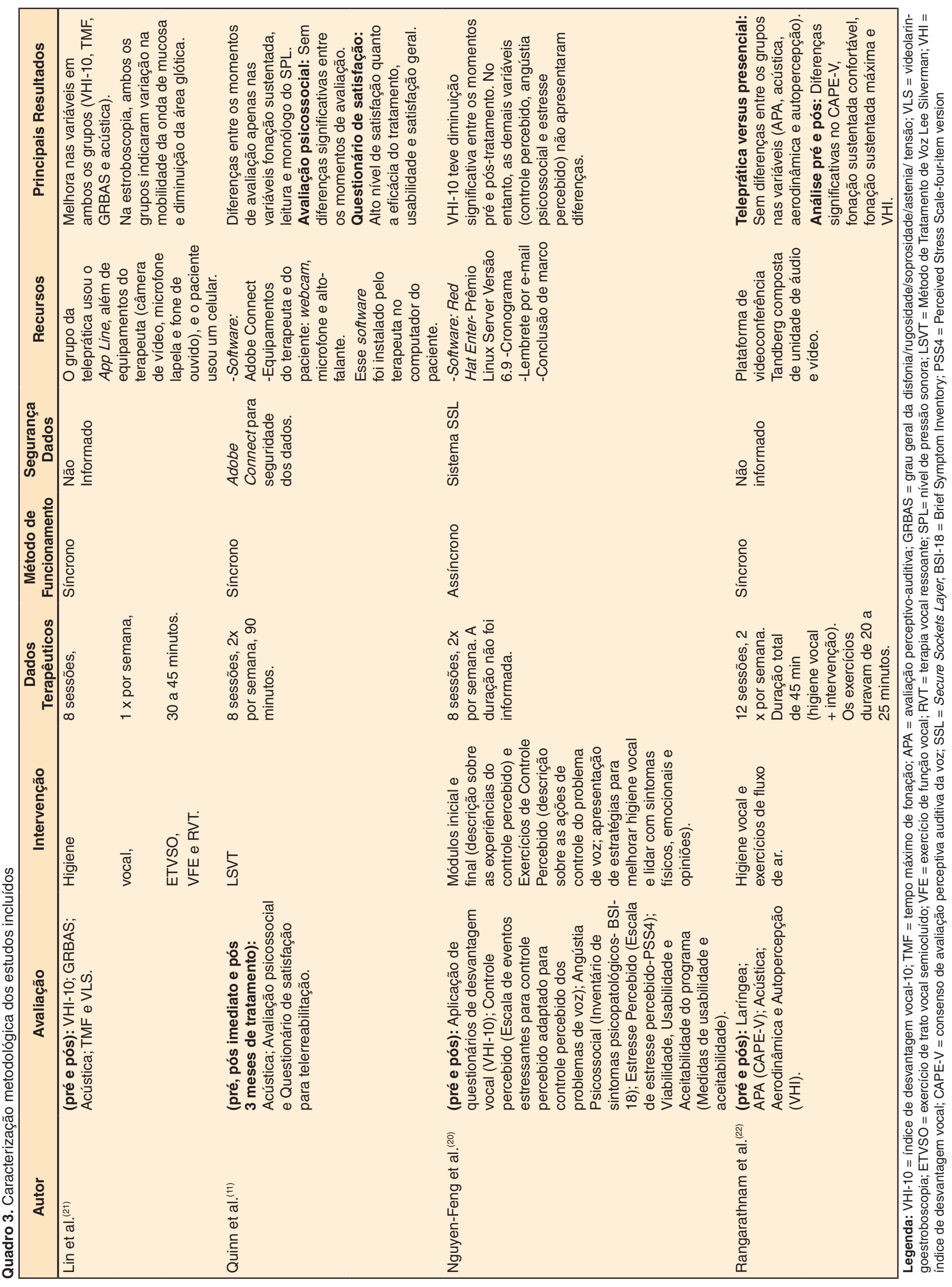




\section{DISCUSSÃO}

O objetivo desta revisão integrativa foi descrever as características da terapia vocal em disfônicos, por meio da Telefonoaudiologia. Após a análise dos artigos, foi possível constatar que a Telefonoaudiologia é atualmente empregada na clínica vocal junto a diferentes públicos com alterações vocais, por meio de recursos variados, que resultaram na satisfação do usuário.

No âmbito da saúde, existe uma gama de termos que estão relacionados aos atendimentos a distância, a saber: telemedicina ${ }^{(23)}$, telessaúde ${ }^{(23)}$, telerreabilitação ${ }^{(24)}$, teleprática ${ }^{(22)}$ e teleatendimento ${ }^{(25)}$. $\mathrm{Na}$ Fonoaudiologia, diferentes órgãos utilizam expressões diversas. No Brasil, o Conselho Federal de Fonoaudiologia (CFFA) adotou, em 2013, telessaúde ${ }^{(4)}$, porém, em 2020, substituiu para Telefonoaudiologia ${ }^{(7)}$. Nos Estados Unidos, a American Speech-Language-Hearing Association (ASHA) adota teleaudiology, telespeech, speech teletherapy e telepractice ${ }^{(26)}$; no Reino Unido, o Royal College of Speech and Language Therapists (RCSLT) usa telehealth ${ }^{(27)}$. Dessa forma, diante da diversidade de terminologias nacionais e internacionais, a presente revisão optou por Telefonoaudiologia, por ser um conceito atual utilizado e adotado pelo Conselho Federal de Fonoaudiologia( ${ }^{(7)}$.

Além do conceito, outro ponto importante é o método de funcionamento do atendimento a distância, que pode ser classificado em assíncrono, síncrono e híbrido ${ }^{(7,26)}$. O assíncrono é o formato em que a interação entre os participantes ocorre de forma offline e de acordo com a disponibilidade de tempo dos sujeitos. Neste modelo, é feito o armazenamento dos arquivos, porém, o acesso é realizado posteriormente pelo paciente ou pelo profissional. Podem ser utilizadas gravação de voz, tarefas de áudio ou vídeo, planejamento de práticas dos exercícios, orientações de higiene vocal, avaliação de medidas acústicas, aerodinâmicas e perceptivo-auditivas ${ }^{(26,28)}$. Já no segundo modelo, o síncrono, o contato entre os participantes ocorre em tempo real e a interação se dá por meio de áudio e vídeo na videoconferência. Neste modelo, também pode ser utilizado o compartilhamento de aplicativos ${ }^{(7,26)}$. Por último, o híbrido destaca-se pela combinação de ambos os formatos, síncrono e assíncrono ${ }^{(7,24)}$. Na presente revisão, o formato predominante foi o síncrono ${ }^{(11,21,22)}$. Apenas um estudo apresentou funcionamento assíncrono $^{(20)}$. De fato, o modelo síncrono reforça a possibilidade de realizar o atendimento online ao vivo com os pacientes, a fim de melhor acompanhar o seu desempenho na reabilitação.

Ressalta-se a realização de serviços voltados para diferentes focos de atuação, como diagnóstico, terapia, monitoramento terapêutico, consultoria, educação e segunda opinião formativa (análise sintética das melhores evidências, que visam auxiliar os profissionais diante da ocorrência dos problemas) $)^{(4,7,8,29-31)}$. A presente revisão, no entanto, teve como foco a investigação do processo terapêutico na área da voz.

Sobre os anos das publicações, chama-se a atenção para a linearidade das pesquisas publicadas nos últimos dez anos, principalmente a predominância dos trabalhos realizados em $2020^{(20,21)}$. Tais realidades demonstram o interesse dos autores pelo aprofundamento e investigação dessa temática no campo da voz, nos dias atuais. Além disso, revelam que, no ano de 2020, fez-se necessário dar continuidade com as pesquisas e com o processo terapêutico por meio da modalidade à distância, como forma de adequação diante da nova realidade de saúde mundial, a pandemia da Covid-19. Cabe ressaltar que, diante do período pandêmico, houve um incremento de pesquisas na área da voz em Telefonoaudiologia, o que permite a comparação entre essas duas modalidades terapêuticas, a fim de conhecer, com maior aprofundamento, a efetividade desses serviços.

Quanto à origem dos atendimentos a distância na Fonoaudiologia, os primeiros estudos datam da década de $1970^{(32)}$, com objetivo de avaliação e tratamento dos distúrbios da comunicação. A partir dos anos 2000, destaca-se a predominância de pesquisas nas áreas da audiologia, fala e linguagem ${ }^{(8,30,33)}$. Dessa forma, percebe-se que essa modalidade, pouco a pouco, está ganhando espaço na Fonoaudiologia e, assim, atingindo todas as especialidades, como por exemplo, a área da voz.

Na distribuição geográfica, os Estados Unidos evidenciaram-se como país de maior publicação. Tal realidade, provavelmente, está relacionada ao fato do país apresentar um alto desenvolvimento tecnológico, favorecendo, assim, a implantação desse formato aos serviços de saúde. De forma semelhante aos achados do atual estudo, revisões sistemáticas ${ }^{(8,12,33)}$ também encontraram elevadas publicações nos EUA e na Austrália, quanto à Telefonoaudiologia. Os Estados Unidos são pioneiros em pesquisas que exploram o atendimento a distância. Já a Austrália, destaca-se por apontar a necessidade de que os fonoaudiólogos realizem atendimento para pacientes de difícil acesso presencial, considerando as distâncias geográficas do país ${ }^{(32)}$.

Cabe ressaltar que, na presente revisão, não foram encontradas pesquisas da Telefonoaudiologia em voz realizada no Brasil, talvez por falta de investimento de profissionais da área no atendimento a distância. Para reforçar essa realidade, uma revisão sistemática sobre telessaúde no Brasil apontou que as principais publicações foram voltadas para a teleducação. Além disso, as áreas mais pesquisadas no país foram audiologia e fonoaudiologia educacional ${ }^{(30)}$. Isso reitera que, na área da voz, a Telefonoaudiologia ainda é pouco explorada.

Outro ponto de destaque é a segurança de dados. Apenas dois artigos ${ }^{(11,20)}$ mencionam o assunto. Um deles ${ }^{(20)}$ relata a utilização de sistema criptografado SSL (Secure Sockets Layer) entre os participantes e pesquisadores. Já o segundo ${ }^{(11)}$, aplicou o Adobe Connect como ferramenta para garantir seguridade e privacidade das sessões. Diante disso, chama-se a atenção para o cuidado e preocupação dos autores em manter o sigilo dos pacientes, preservando seus dados e sua identidade.

No que se refere ao delineamento dos estudos, observouse uma distribuição igualitária quanto aos estudos quase experimentais e os ensaios clínicos randomizados. Quanto aos trabalhos quase experimentais ${ }^{(11,20)}$, foram investigados programas de intervenções realizadas sem aleatorização. Sobre as pesquisas do tipo ensaio clínico randomizado, destaca-se a necessidade dos autores em comprovar que a utilização da terapia vocal à distância pode ser utilizada no contexto clínico como nova modalidade terapêutica ${ }^{(22)}$ sem apresentar prejuízos ao atendimento presencial ${ }^{(21)}$.

Em relação aos objetivos das pesquisas desta revisão, destacaram-se a aplicação da terapia vocal por Telefonoaudiologia em grupos com diferentes diagnósticos e demandas, como doença de Parkinson ${ }^{(11)}$, disfonia por tensão muscular ${ }^{(20,22)}$ e em idosos ${ }^{(21)}$ com atrofia de prega vocal, paralisia unilateral de prega vocal, disfonia por tensão muscular, nódulos e pólipos. Chama-se a atenção para a aplicação da Telefonoaudiologia em pacientes com disfonia por tensão muscular, pela dificuldade de realizar palpação laríngea na reabilitação virtual, porém, levanta-se a possibilidade de que esse público também possa ser beneficiado, 
apesar de não ser trabalhado o contato corporal nessa nova modalidade. Outros estudos realizaram de forma predominante na população com disfonia neurogênica, principalmente em indivíduos com Parkinson ${ }^{(10,12,15,32,34)}$. Além dessa etiologia, também foram encontrados nódulos vocais ${ }^{(14,35)}$, disartria ${ }^{(36)}$, edema laríngeo $^{(35)}$ e disfonia por tensão muscular ${ }^{(35)}$. Dessa forma, tal realidade é exposta em um estudo ${ }^{(34)}$, ao descobrir que apenas 3\% na área da voz utilizam a reabilitação da teleprática na Fonoaudiologia - o que indica a necessidade de ampliar as pesquisas nesse formato.

Quanto aos dados dos participantes - sexo, faixa etária e tamanho da amostra -, observou-se que, na maioria dos estudos, predominavam as mulheres ${ }^{(20-22)}$. Apenas em um estudo ${ }^{(11)}$ o número de participantes do sexo masculino foi destaque, em relação ao sexo feminino. Ao contrário dos resultados apresentados nesta revisão, é possível encontrar na literatura em Fonoaudiologia uma inconsistência em relação ao sexo pesquisado. Alguns não investigam o sexo dos participantes ${ }^{(10,12,33,34)}$, enquanto outros demonstram predominância feminina ${ }^{(14)}$ ou masculina ${ }^{(15,35)}$. Diante de tal variação, é possível que o sexo não seja uma variável determinante na opção por Telefonoaudiologia. Estudos futuros são necessários para esclarecer tal questão.

A faixa etária predominante dos sujeitos pesquisados incluiu o público idoso e variou de 60 a 82 anos. Cabe destacar a relevância desse achado, especialmente ao verificar que os estudos ${ }^{(20,21)}$ publicados no ano de 2020 foram realizados com uma população considerada de alto risco para o novo coronavírus. Além disso, nesta revisão, observou-se que dois estudos apresentaram faixas etárias de jovem ao idoso ${ }^{(20,22)}$, enquanto uma pesquisa variou de adulto ao idoso ${ }^{(21)}$. Ressalta-se, no entanto, que a literatura aponta um estudo que aborda terapia vocal em 28 disfônicos de 6 a 18 anos, que participaram da reabilitação com vídeos em MP4 ${ }^{(35)}$. Isso retrata que a Telefonoaudiologia na voz pode ser empregada em todas as faixas etárias, desde a infância até a senescência. Na presente revisão, entretanto, foram encontrados artigos que abrangeram o público jovem, adulto e idoso, de acordo com os critérios de elegibilidade e avaliação crítica estabelecidos.

Sobre o tamanho das amostras, os trabalhos incluídos indicaram variações, no entanto, a maioria das publicações realizou atendimentos em grupos ${ }^{(20-22)}$, sendo apenas uma na modalidade individual ${ }^{(11)}$. No que se refere ao quantitativo total de participantes, as publicações apontaram para uma variação de oito a 69 sujeitos $^{(11,20-22)}$, quantidade que está na faixa de participantes de outros estudos sobre Telefonoaudiologia em Fonoaudiologia, que varia de dois a $100^{(33,34)}$. Na área de voz, as pesquisas apontam uma variação de dez a $51^{(10,14,15,28,35,37)}$. Tal discrepância pode estar relacionada às variações metodológicas dos estudos realizados em Telefonoaudiologia.

Quanto às modalidades terapêuticas, duas pesquisas desta revisão realizaram comparações entre atendimento presencial e Telefonoaudiologia ${ }^{(21,22)}$ e não encontraram diferenças entre os parâmetros vocais (acústica, perceptivo-auditiva, TMF, aerodinâmica e autopercepção). Isso pode indicar que a Telefonoaudiologia não é inferior à terapia tradicional, mas possui semelhança quanto aos resultados terapêuticos, mostrando, assim, que pode ser aplicada na clínica vocal como um novo formato de intervenção. Tal realidade também é encontrada na literatura, ao verificar ganhos em parâmetros vocais acústicos e perceptivo-auditivos, assim como na terapia presencial ${ }^{(37)}$. Se analisada pela esfera atual, destaca-se que a aplicabilidade desse formato a distância pode ser viável, especialmente no período da pandemia. Estudo nacional ${ }^{(38)}$ apontou que o atendimento presencial realizado em uma unidade básica, com duração de 30 minutos, no período da pandemia, passou a ser empregado como Telefonoaudiologia, com variação de 20 a 60 minutos, de acordo com o caso do paciente. Vale ressaltar que, no Brasil, não existem estudos comparativos entre as modalidades terapêuticas. Considerando o momento pandêmico, faz-se necessário a realização de trabalhos que possam fazer tal análise, a fim de conhecer as diferentes realidades terapêuticas vivenciadas nesse novo contexto de saúde.

A duração dos atendimentos, na maioria dos estudos, foi de oito sessões ${ }^{(11,18,21)}$, duas vezes por semana ${ }^{(11,20,22)}$. No entanto, o tempo de execução da terapia variou de 30 a 90 minutos $^{(11,20-22)}$. $\mathrm{Na}$ literatura, as metodologias são apresentadas de forma diferente no que se refere à quantidade de sessões: nove a 18 sessões $^{(10,15,34,39)}$; frequência de três vezes por semana ${ }^{(14,15)}$ e quatro vezes por semana ${ }^{(10,37)}$; duração de uma hora ${ }^{(10,37,38)}$, ou 30 minutos $^{(15)}$, ou 45 minutos $^{(14)}$. Essas realidades também expressam falta de padronização terapêutica.

No processo de intervenção vocal, destacou-se a realização de higiene vocal ${ }^{(20-22)}$ como medida de orientação vocal antes da aplicação dos exercícios terapêuticos. Sobre esses exercícios, ressalta-se uma variedade em cada estudo, expressando melhora entre os parâmetros vocais no pós-treinamento (autopercepção vocal, TMF, aerodinâmico, perceptivo-auditiva e acústica) $)^{(11,20-}$ ${ }^{22)}$, além de satisfação do usuário com a intervenção online ${ }^{(11)}$ e aceitabilidade e usabilidade quanto ao programa ${ }^{(20)}$. Tais evidências apontam para a efetividade dessa modalidade de atendimento na área de voz. Chama-se a atenção para uma possibilidade terapêutica que mantém os mesmos ganhos da terapia presencial no ambiente virtual.

Na presente revisão, salientaram-se duas pesquisas ${ }^{(11,21)}$ que apontaram para realização de exercícios em casa, como forma de continuidade da reabilitação vocal. Em um dos estudos ${ }^{(21)}$, realizava-se entrega de ilustrações referentes às orientações de higiene vocal, às tarefas do exercício de função vocal (VFE) e da terapia vocal ressoante. Além disso, era utilizada uma ficha de acompanhamento sobre a realização dos exercícios em casa, para facilitar ofeedback. O outro estudo ${ }^{(11)}$ mostrou a solicitação de exercícios em domicílio quanto às tarefas fonatórias, leitura e conversação em voz alta. Os pesquisadores faziam sempre a revisão das atividades realizadas em casa no início de cada sessão terapêutica. Essas duas pesquisas indicam que, mesmo com os atendimentos a distância, é possível manter o monitoramento da terapia vocal, com a solicitação e acompanhamento da execução de exercícios em casa.

Os recursos usados na maioria das pesquisas foram softwares $^{(11,20)}$, aplicativos ${ }^{(21)}$ e plataforma de videoconferência ${ }^{(22)}$. Além disso, outros recursos foram utilizados para apresentar uma boa qualidade quanto ao vídeo e a imagem, a saber: câmera de vídeo, ou webcam, microfone, fone de ouvido e alto-falante. Outro ponto de destaque foi a necessidade do terapeuta ir ao domicílio do paciente instalar o software ${ }^{(11)}$ ou testar a internet ${ }^{(21)}$ antes da intervenção. No entanto, apenas um estudo $^{(22)}$ informou que uma equipe estava disponível, caso o usuário tivesse dúvidas. Esses últimos dados apontam para a necessidade de um ambiente favorável para o bom funcionamento dessa modalidade virtual, seja por meio dos equipamentos ou de um suporte técnico.

Por último, cabe ressaltar que os estudos selecionados nesta revisão foram publicações oriundas dos Estados Unidos, da Austrália e da China. Neste sentido, as abordagens no 
atendimento a distância no Brasil podem divergir em decorrência das diferenças nos aspectos culturais e socioeconômicos. No Brasil, as publicações no campo da Telefonoaudiologia têm foco na teleducação, por meio de medidas de orientação vocal $^{(13,30)}$ e também, com o exercício da teleconsultoria ${ }^{(31,38)}$ e do telemonitoramento terapêutico ${ }^{(38)}$. Com o advento da pandemia da Covid-19, é possível que as futuras publicações apresentem o perfil de atendimento remoto mais próximo da realidade dos países apontados.

As limitações desta revisão foram a quantidade reduzida de pesquisas de Telefonoaudiologia na área da voz e a falta de padronização metodológica nas intervenções virtuais. Assim, evidencia-se a necessidade de mais estudos com alto rigor metodológico em Telefonoaudiologia em voz, para dar embasamento teórico aos profissionais, a fim de aperfeiçoar os atendimentos com as Tecnologias da Informação e Comunicação (TICs). Salienta-se, além disso, a importância de desenvolver novas pesquisas no Brasil, tendo em vista que a diversidade cultural do país propicia um novo olhar para esse campo. Aponta-se, também, a necessidade de desenvolver trabalhos comparando o atendimento presencial e a Telefonoaudiologia no Brasil, para que seja possível compreender as diferenças dessas modalidades na realidade nacional. Por fim, sugere-se a criação de protocolos para Telefonoaudiologia em voz, a fim de permitir a padronização nas pesquisas e nas metodologias dos estudos futuros.

\section{CONCLUSÃO}

A Telefonoaudiologia junto a pacientes disfônicos é voltada para diferentes públicos com etiologias vocais diversas, a saber: doença de Parkinson; disfonia por tensão muscular e idosos com alterações vocais. Além disso, para o atendimento em voz a distância, há diferentes metodologias empregadas, a exemplo de amostra, método de funcionamento, segurança de dados, número de sessões, frequência semanal, duração da intervenção e tipos de exercícios aplicados - com registro de melhorias nos parâmetros vocais (acústicos, perceptivo-auditivos, TMF, aerodinâmicos e autopercepção), bem como satisfação do usuário sobre o tratamento nesse formato.

Dessa forma, a intervenção a distância na área da voz coloca-se como uma possibilidade promissora de modalidade de atendimento. Destaca-se a necessidade de formação de profissionais para a Telefonoaudiologia, considerando-se, inclusive, possíveis soluções para as barreiras que se fazem presentes.

\section{AGRADECIMENTOS}

À Coordenação de Aperfeiçoamento de Pessoal de Nível Superior (CAPES) pelo financiamento desta pesquisa de pósgraduação na modalidade de mestrado.

\section{REFERÊNCIAS}

1. Dunkley C, Pattie L, Wilson L, McAllister L. A comparison of rural speech-language pathologists' and residents' access to and attitudes towards the use of technology for speech-language pathology service delivery. Int J Speech Lang Pathol. 2010 Ago;12(4):333-43. http:// dx.doi.org/10.3109/17549500903456607. PMid:20590518.

2. Eslami Jahromi M, Ahmadian L. Evaluating satisfaction of patients with stutter regarding the tele-speech therapy method and infrastructure. Int J Med Inform. 2018 Jul;115:128-33. http://dx.doi.org/10.1016/j. ijmedinf.2018.03.004. PMid:29779715.

3. Hart J. Expanding access to telespeech in clinical settings: inroads and challenges. Telemed J E Health. 2010 Nov;16(9):922-4. http:// dx.doi.org/10.1089/tmj.2010.9945. PMid:21091285.

4. Brasil. Conselho Federal de Fonoaudiologia. Resolução no ${ }^{\circ} 427$, de 1 de março de 2013. Dispõe sobre a regulamentação da telessaúde em Fonoaudiologia e dá outras providências. Diário Oficial da União; Brasília; 5 mar. 2013.

5. Mattei A, Amy de la Bretèque B, Crestani S, Crevier-Buchman L, Galant C, Hans S, et al. Guidelines of clinical practice for the management of swallowing disorders and recent dysphonia in the context of the COVID-19 pandemic. Eur Ann Otorhinolaryngol Head Neck Dis. 2020 Maio;137(3):173-5. http://dx.doi.org/10.1016/j.anorl.2020.04.011. PMid:32332004.

6. Castillo-Allendes A, Contreras-Ruston F, Cantor-Cutiva LC, Codino J, Guzman M, Malebran C, et al. Voice therapy in the context of the COVID-19 pandemic: guidelines for clinical practice. J Voice. 2021 Sep;35(5):717-27. http://dx.doi.org/10.1016/j.jvoice.2020.08.001. PMid:32878736.

7. Brasil. Conselho Federal de Fonoaudiologia. Resolução n ${ }^{\circ} 580$, de 20 de agosto de 2020. Dispõe sobre a regulamentação da telefonoaudiologia e dá outras providências. Diário Oficial da União; Brasília; 25 ago. 2020 .

8. Molini-Avejonas DR, Rondon-Melo S, Amato CA, Samelli AG. A systematic review of the use of telehealth in speech, language and hearing sciences. J Telemed Telecare. 2015 Out;21(7):367-76. http:// dx.doi.org/10.1177/1357633X15583215. PMid:26026181.

9. Keck CS, Doarn CR. Telehealth technology applications in speechlanguage pathology. Telemed J E Health. 2014 Jul;20(7):653-9. http:// dx.doi.org/10.1089/tmj.2013.0295. PMid:24820794.

10. Griffin M, Bentley J, Shanks J, Wood C. The effectiveness of Lee Silverman Voice Treatment therapy issued interactively through an iPad device: a non-inferiority study. J Telemed Telecare. 2018;24(3):20915. http://dx.doi.org/10.1177/1357633X17691865. PMid:28147896.

11. Quinn R, Park S, Theodoros D, Hill AJ. Delivering group speech maintenance therapy via telerehabilitation to people with Parkinson's disease: A pilot study. Int J Speech Lang Pathol. 2019;21(4):385-94. http://dx.doi.org/10.1080/17549507.2018.1476918. PMid:29879854.

12. Theodoros D, Aldridge D, Hill AJ, Russell T. Technology-enabled management of communication and swallowing disorders in Parkinson's disease: a systematic scoping review. Int J Lang Commun Disord. 2019 Mar;54(2):170-88. http://dx.doi.org/10.1111/1460-6984.12400. PMid:29923267.

13. Oliveira LF, Corrêa CC, Vieira MMRM, Blasca WQ, Brasolotto AG. Intervention via teleducation about vocal mutation and vocal habits. Audiol Commun Res. 2018;23:e1899. http://dx.doi.org/10.1590/23176431-2017-1899.

14. Fu S, Theodoros DG, Ward EC. Delivery of intensive voice therapy for vocal fold nodules via telepractice: a pilot feasibility and efficacy study. J Voice. 2015 Nov;29(6):696-706. http://dx.doi.org/10.1016/j. jvoice.2014.12.003. PMid:25726070.

15. Chan MY, Chu SY, Ahmad K, Ibrahim NM. Voice therapy for Parkinson's disease via smartphone videoconference in Malaysia: a preliminary 
study. J Telemed Telecare. J Telemed Telecare. 2021 Apr;27(3):17482. http://dx.doi.org/10.1177/1357633X19870913. PMid:31431134.

16. Mendes KDS, Silveira RCCP, Galvão CM. Revisão integrativa: método de pesquisa para a incorporação de evidências na saúde e na enfermagem. Texto Contexto Enferm. 2008;17(4):758-64. http:// dx.doi.org/10.1590/S0104-07072008000400018.

17. JBI: Joanna Briggs Institute. Joanna Briggs Institute reviewers' manual 2015 edition: methodology for JBI scoping reviews [Internet]. Adelaide: The Joanna Briggs Institute; 2015 [citado em 2021 Out 12]. Disponível em: https://nursing.lsuhsc.edu/JBI/docs/ReviewersManuals/Scoping-. pdf

18. JBI: Joanna Briggs Institute. Critical appraisal tools [Internet]. Adelaide: The Joanna Briggs Institute; 2021 [citado em 2021 Mar 4]. Disponível em: https://jbi.global/critical-appraisal-tools

19. Trindade CS, Ramos AL. Influência dos programas de educação sobre o sono de crianças e adolescentes: revisão integrativa. Acta Paul Enferm. 2020;33:1-9. http://dx.doi.org/10.37689/acta-ape/2020AR01936.

20. Nguyen-Feng VN, Frazier PA, Stockness A, Narayanan A, Merians AN, Misono S. Web-based perceived present control intervention for voice disorders: a pilot study. J Voice. 2020 Mar;34(2):300.e1-9. http://dx.doi.org/10.1016/j.jvoice.2018.08.006. PMid:30227981.

21. Lin FC, Chien HY, Chen SH, Kao YC, Cheng PW, Wang CT. Voice therapy for benign voice disorders in the elderly: a randomized controlled trial comparing telepractice and conventional face-to-face therapy. J Speech Lang Hear Res. 2020;63(7):2132-40. http://dx.doi. org/10.1044/2020_JSLHR-19-00364. PMid:32579859.

22. Rangarathnam B, McCullough GH, Pickett H, Zraick RI, TulunayUgur O, McCullough KC. Telepractice versus in-person delivery of voice therapy for primary muscle tension dysphonia. Am J Speech Lang Pathol. 2015 Ago;24(3):386-99. http://dx.doi.org/10.1044/2015 AJSLP-14-0017. PMid:25836732.

23. Weinstein RS, Krupinski EA, Doarn CR. Clinical examination component of Telemedicine, Telehealth, mHealth, and connected health medical practices. Med Clin North Am. 2018 Maio;102(3):533-44. http://dx.doi.org/10.1016/j.mcna.2018.01.002. PMid:29650074.

24. Choon-Huat Koh G, Hoenig H. How should the rehabilitation community prepare for 2019-nCoV? Arch Phys Med Rehabil. 2020 Jun;101(6):1068-71. http://dx.doi.org/10.1016/j.apmr.2020.03.003. PMid:32194034.

25. Araújo ADIR, Arruda LSNS. Tele-service as monitoring tool for suspected and/or confirmed cases of COVID-19. Braz J Develop. 2020;6(8):57807-15.

26. Grillo EU. Building a successful voice telepractice program. Perspect ASHA Spec Interest Groups. 2019 Fev;4(1):100-10. http://dx.doi. org/10.1044/2018_PERS-SIG3-2018-0014. PMid:31544152.

27. RCSLT: Royal College of Speech and Language Therapists. COVID-19 speech and language therapy rehabilitation pathway [Internet]. London: RCSLT; 2020 [citado em 2020 Jul 14]. Disponível em: https://www.
rcslt.org/wp-content/uploads/media/docs/Covid/RCSLT-COVID-19SLT-rehab-pathway_15-July-2020_FINAL.pdf

28. Grillo EU. An online telepractice model for the prevention of voice disorders in vocally healthy student teachers evaluated by a smartphone application. Perspect ASHA Spec Interest Groups. 2017 Jun;2(3):6378. http://dx.doi.org/10.1044/persp2.SIG3.63. PMid:28890933.

29. Nascimento CMB, et al. Telespeech therapy as a continued education strategy in primary health care in the state of Pernambuco, Brazil. Rev CEFAC. 2017 Maio-Jun;19(3):371-80. http://dx.doi.org/10.1590/19820216201719314716 .

30. Fonsêca RO, Brazorotto JS, Balen AS. Telehealth use in speech, language and hearing pathology in Brazil: systematic review. Rev CEFAC. 2015 Nov-Dez;17(6):2033-43.

31. Barros VV, et al. An analysis of asynchronous hearing health teleconsulting at the Rio Grande do Norte Telehealth Center. Audiol Commun Res. 2021;26:e24.

32. Mashima PA, Brown JE. Remote management of voice and swallowing disorders. Otolaryngol Clin North Am. 2011 Dec;44(6):1305-16, viii. http://dx.doi.org/10.1016/j.otc.2011.08.007. PMid:22032484.

33. Wales D, Skinner L, Hayman M. The efficacy of telehealth-delivered speech and language intervention for primary school-age children: a systematic review. Int J Telerehabil. 2017 Jun 29;9(1):55-70. http:// dx.doi.org/10.5195/ijt.2017.6219. PMid:28814995.

34. Weidner K, Lowman J. Telepractice for adult speech-language pathology services: a systematic review. Perspect ASHA Spec Interest Groups. 2020;5(1):326-38. http://dx.doi.org/10.1044/2019_PERSP-19-00146.

35. Braden MN, van Leer E. Effect of MP4 therapy videos on adherence to voice therapy home practice in children with dysphonia. J Voice. 2017 Jan;31(1):114.e17-23. http://dx.doi.org/10.1016/j.jvoice.2016.03.015. PMid:27133000.

36. Bakker M, Beijer L, Rietveld T. Considerations on effective feedback in computerized speech training for dysarthric speakers. Telemed J E Health. 2019 Maio;25(5):351-8. http://dx.doi.org/10.1089/tmj.2018.0050. PMid:30074851.

37. Constantinescu G, Theodoros D, Russell T, Ward E, Wilson S, Wootton $\mathrm{R}$. Treating disordered speech and voice in Parkinson's disease online: a randomized controlled non-inferiority trial. Int J Lang Commun Disord. 2011 Jan-Fev;46(1):1-16. http://dx.doi.org/10.3109/136828 22.2010.484848. PMid:21281410.

38. Dimer NA, Canto-Soares ND, Santos-Teixeira LD, Goulart BNG. The COVID-19 pandemic and the implementation of telehealth in speech-language and hearing therapy for patients at home: an experience report. CoDAS. 2020;32(3):e20200144. http://dx.doi. org/10.1590/2317-1782/20192020144. PMid:32578694.

39. Botton A, Cúnico SD, Strey MN. Diferenças de gênero no acesso aos serviços de saúde: problematizações necessárias. Mudanças Psicol Saúde. 2017;25(1):67-72. http://dx.doi.org/10.15603/2176-1019/mud. v25n1p67-72. 